\title{
Incidence of long-term cardiotoxicity and evolution of the systolic function in patients with breast cancer treated with anthracyclines
}

\author{
Rebeca Mata Caballero ${ }^{1}$, José María Serrano Antolín², Rosa María Jiménez Hernández², \\ Pedro Talavera Calle ${ }^{2}$, Alejandro Curcio Ruigómez ${ }^{2}$, Silvia del Castillo Arrojo², \\ Catherine Graupner $\mathrm{Abad}^{2}$, Carmen Cristóbal Varela ${ }^{2}$, Joaquín Jesús Alonso Martín ${ }^{1}$ \\ ${ }^{1}$ Cardiology Department, Hospital Universitario de Getafe, Madrid, Spain \\ ${ }^{2}$ Cardiology Department, Hospital Universitario de Fuenlabrada, Madrid, Spain
}

\begin{abstract}
Background: Anthracycline cardiotoxicity (AC) may manifest years after treatment (long-term cardiotoxicity). There is little data on the incidence and natural history of $A C$ in the current context, with protocols including lower anthracycline doses. The present study prospectively evaluated the incidence, time of occurrence and clinical correlates of long-term cardiotoxicity and the evolution of systolic function in patients with breast cancer treated with anthracyclines.

Methods: This study prospectively included 85 consecutive patients undergoing chemotherapy (CHT) with anthracyclines without trastuzumab. All patients underwent evaluation at baseline, at the end of CHT, 3 months after the end of CHT and 1 and 4 years subsequent to the beginning of CHT. Clinical data and echocardiographic parameters were evaluated in all examinations.

Results: The mean dose of doxorubicin used was $243.53 \mathrm{mg} / \mathrm{m}^{2}$. Median follow-up of the current cohort was 4.5 years. At 1 year the incidence of $A C$ was $1 \%$ and at the end of the follow-up 16.5\% (14 of 85 patients). Therefore, the incidence of late cardiotoxicity (after the first year) was 15\%. Of these 14 patients with AC, 12 had asymptomatic systolic dysfunction, 1 had heart failure and 1 suffered sudden death. Fifteen percent developed systolic dysfunction during follow-up. An early decline in strain was observed in patients who developed long-term AC.

Conclusions: The incidence of long-term cardiotoxicity in patients treated with low-cumulative dose of anthracyclines is high, $16.5 \%$ at 4.5 years. This was observed in almost all cases after the first year of follow-up. Therefore, long-term monitoring may be advisable. (Cardiol J 2022; 29, 2: 228-234)
\end{abstract}

Key words: cardiotoxicity, anthracyclines, cardiomyopathies, drug therapy, heart failure, systolic dysfunction

\section{Introduction}

Anthracyclines are highly effective chemotherapy (CHT) agents of a broad spectrum widely used in cancer processes for diseases such as breast cancer, leukemias and lymphomas. However, the utility of anthracyclines is limited by cumulative, dose-related, progressive myocardial damage that may lead to congestive heart failure [1-3]. Anthracycline cardiotoxicity (AC), which may manifest years after treatment (long-term cardiotoxicity) $[4,5]$.

There are discordant data regarding the incidence of AC. Most of the available data come

Address for correspondence: Rebeca Mata Caballero, Cardiology Department, Hospital Universitario de Getafe, Carretera de Toledo Km 12,500, 28905 - Getafe (Madrid), Spain, tel: +34 680556895, fax: +34 916247313, e-mail: rebecamca@gmail.com 
from retrospective studies, with substantial variability in the incidence of AC, depending on the definition of cardiotoxicity, the cumulative dose of anthracyclines, the age of the patients and the drugs used. AC reported ranges from $5 \%$, when a cumulative lifetime dose of $400 \mathrm{mg} / \mathrm{m}^{2}$ is reached, up to $48 \%$ at $700 \mathrm{mg} / \mathrm{m}^{2}$ [6-8], when higher doses lead to an exponential increase in risk. In addition, it was found that increasing age was associated with an increasing risk of $\mathrm{AC}[9,10]$. These incidences reported of AC may not be an accurate reflection of the true incidence nowadays, where regimens are including lower anthracycline doses and an initial cardiological evaluation is attempted to exclude patients with underlying heart disease. Early detection and prompt therapy of cardiotoxicity appear crucial for substantial recovery of cardiac function [11].

The aim of the study was to evaluate the incidence of long-term cardiotoxicity and the evolution of systolic function during the follow-up, in patients with breast cancer treated with anthracyclines without trastuzumab in the current context, excluding patients with previous heart disease.

\section{Methods}

\section{Study population}

This prospective and analytical cohort study was conducted in the area of Hospital of Fuenlabrada, Madrid. All consecutive patients were considered who had breast cancer treated with anthracycline-based CHT (adjuvant and neoadjuvant therapy) during the period from April 2008 to May 2010, whose age was between 18 and 80 years.

Patients treated with anthracycline therapy followed by trastuzumab were excluded.

Patients were also excluded if they had had a previous cardiac disease (ischemic heart disease, moderate or severe valvular heart disease, dilated, restrictive or hypertrophic cardiomyopathy), history of heart failure or left ventricular ejection fraction $(\mathrm{LVEF})<55 \%$; patients with a poor echocardiographic window, permanent atrial fibrillation, renal insufficiency with creatinine $>2 \mathrm{mg} / \mathrm{dL}$ or clearance $<30 \mathrm{~mL} / \mathrm{h}$, previous treatment with anthracyclines or a life expectancy of $\leq 1$ year were also excluded.

The local Ethical Committee approved the study, and all patients provided written informed consent.

\section{Study protocol}

Patients in the study received one of two different CHT regimens: FECX6 (Group 1): 5-fluoruracil $600 \mathrm{mg} / \mathrm{m}^{2}$, epirubicin $75 \mathrm{mg} / \mathrm{m}^{2}$ and cyclophosphamide $600 \mathrm{mg} / \mathrm{m}^{2}, 1$ cycle every 21 days until 6 cycles were completed; ACX4-T (Group 2): doxorubicin $60 \mathrm{mg} / \mathrm{m}^{2}$ and cyclophosphamide $600 \mathrm{mg} / \mathrm{m}^{2}, 1$ cycle every 21 days to complete 4 cycles followed by paclitaxel (taxol) $80 \mathrm{mg} / \mathrm{m}^{2}$, 1 weekly cycle for 12 weeks.

Patients were evaluated on five separate visits: - before the start of anthracycline therapy (visit 0);

- just prior to the last dose of anthracycline CHT (visit 1);

- 3 months after the last dose of anthracycline CHT (visit 2);

- 9 months after the last dose of anthracycline CHT (visit 3);

- 4 years after the start of the CHT (visit 4).

In the group of patients who received an epirubicin dose, estimated by using a conversion factor of 0.55 (50 $\mathrm{mg}$ of doxorubicin $=90 \mathrm{mg}$ of epirubicin), according to the recommendations of the Oncology Department and published data [12].

Follow-up visits were adapted to previous knowledge of chronic anthracycline cardiomyopathy $[7,11,13-15]$. These time points were adapted to planned oncological controls, for ethical concerns and to humanize the research.

On each visit clinical status, physical examination, as well as signs and symptoms of heart failure were evaluated. An electrocardiogram and a complete echocardiogram were performed, including measurement of LVEF (BIPLANE method) [14] and global longitudinal strain (GLS). Echocardiograms were performed utilizing GE Vivid Cardiac Ultrasound (General Electric, Milwaukee, USA), then digitized and analyzed using EchoPAC software (GE medical systems, Milwaukee, USA).

\section{Study endpoints}

The aim of the study was to evaluate the incidence of long-term cardiotoxicity in patients with breast cancer treated with anthracyclines. According to the definition of cardiotoxicity accepted at the time when the study began [16], AC was defined as new-onset heart failure, according to the Framingham criteria, reduction in LVEF $\geq 10$ percentage points from baseline with a final LVEF less than $55 \%$, sustained ventricular arrhythmias, or sudden cardiac death.

Cardiotoxicity was classified in different types depending on the time of appearance: acute cardiotoxicity, immediately after the CHT dose; chronic cardiotoxicity, within the first year after the start of CHT; and late cardiotoxicity which occurs later than a year after CHT has been completed [17-20]. Long-term cardiotoxicity includes chronic cardiotoxicity and late cardiotoxicity. 
The secondary endpoints were to assess the evolution of the left systolic function in these patients, in order to establish their role in the early diagnosis of cardiotoxicity.

\section{Statistical analyses}

Statistical analyses were performed using the Statistical Package for Social Sciences (SPSS), version 15.0 for Windows. Data are summarized as mean with standard deviation, or proportions, as in the case of discrete variables. The Paired Student t-test was used for comparisons of continuous variables between follow-up and baseline visits. Univariate comparisons of patient groups who developed anthracycline cardiotoxicity $(\mathrm{AC}+)$ or those who did not (AC-), were analyzed by means of the Student's t-test or the Fisher exact test.

\section{Results}

Eighty-five patients (98\% women, mean age: 50.4 years) were included in the study. The mean dose of doxorubicin used was $243.53 \mathrm{mg} / \mathrm{m}^{2}$ of body surface area. The mean follow-up of the present cohort was 52 months ( $52 \pm 8.2$ months). Baseline characteristics of the patients are shown in Table 1 . Ninety percent of the population received radiotherapy but only $42 \%$ (36 patients) received radiotherapy on the left hemithorax or mediastinum. Mean radiotherapy total dose was $5916.13 \mathrm{~Gy}$. Regarding heart dose, heart volume that received 30 Gy of radiotherapy (V30 Gy) was 6.3\%.

At 1 year of follow-up, 2 patients died, one due to sudden cardiac death (attributed to cardiotoxicity) and the other due to progression of the oncological disease. At the end of follow-up, 8 of the 85 patients had died (9.4\%). Seventy-five patients attended all scheduled visits. Visit 3 was attended by $99 \%$ of patients alive at that time and last visit was attended by $100 \%$ of patients alive at that time.

During the first year of follow-up, 1 patient developed AC (1\%). After the first year, another 13 patients developed cardiotoxicity (late cardiotoxicity). Therefore, at the end of the follow-up, 14 patients had developed AC (16.5\%) (longterm cardiotoxicity), of which: 1 patient had heart failure and left ventricular systolic dysfunction, 12 patients had asymptomatic left ventricular systolic dysfunction and 1 patient suffered sudden cardiac death.

The distribution according to the CHT schemes was: 6 patients from the FECx6 group (20\% of them) and 8 patients from the ACx4-T group (15\%). Regarding the clinical variables, the
Table 1. Baseline characteristics of the patients.

\begin{tabular}{lc}
\hline Number of patients & 85 \\
Age [years] & $50.4 \pm 9.03$ \\
Female sex & $83(98 \%)$ \\
Weight $[\mathrm{kg}]$ & $69.9 \pm 11.7$ \\
High $[\mathrm{cm}]$ & $1.57 \pm 7.1$ \\
Body mass index $\left[\mathrm{kg} / \mathrm{m}^{2}\right]$ & $28.41 \pm 5.0$ \\
Cardiovascular risk factors: & \\
Hypertension & $26(31 \%)$ \\
Smoking & $29(34 \%)$ \\
Hyperlipidemia & $14(17 \%)$ \\
Diabetes & $10(12 \%)$ \\
Chemotherapy regimen: & \\
FECx6 & $30(35 \%)$ \\
ACx4-T & $55(65 \%)$ \\
Anthracycline dose $\left[\mathrm{mg} / \mathrm{m}^{2}\right]$ & $243.53 \pm 4.8$ \\
Radiotherapy & $36(42 \%)$ \\
Beta-blockers treatment & $2(2 \%)$ \\
ACEl treatment & $13(15 \%)$ \\
\hline
\end{tabular}

Data are shown as mean standard deviation or number (percentage). FEC $\times 6-5$-fluorouracil, epirubicin and cyclophosphamide; ACx4-T - adriamycin (doxorubicin), cyclophosphamide and Taxol ${ }^{\circledR}$ (paclitaxel); ACEI — angiotensin-converting enzyme inhibitors

univariate analysis showed no differences in age ( 50.1 vs. 51.6 years), body mass index or any of the cardiovascular risk factors between the $\mathrm{AC}+$ and $\mathrm{AC}$-groups, as shown in Table 2. There were no significant differences in other clinical variables evaluated as radiotherapy, anthracycline dose or CHT scheme. Neither differences were found in the proportion of patients who were being treated with angiotensin-converting enzyme inhibitors or statins between the groups at the beginning of the study. The use of beta-blockers was greater among $\mathrm{AC}+$ patients, although only 2 patients were being treated with beta-blockers, hence, it was difficult to draw conclusions on this point. The main variables in the group of patients who developed anthracycline cardiotoxicity $(\mathrm{AC}+)$ and those in the group that did not develop anthracycline cardiotoxicity (AC-) are summarized in Table 2.

\section{Echocardiographic findings}

All patients had a normal basal systolic function, according to the inclusion criteria and selection of candidates for treatment with anthracyclines, with a mean LVEF of $67.13 \pm 5.9 \%$ (Table 3 ) and there were no differences between different CHT protocols. Similarly, all patients presented baseline values of normal GLS, with 
Table 2. Main clinical variables and echocardiographic parameters in groups AC+ and AC-.

\begin{tabular}{lccc}
\hline & AC + & AC- & P \\
\hline Number of patients & 14 & 71 & 0.78 \\
Age [years] & $50.1 \pm 9.5$ & $51.6 \pm 9.03$ & 0.87 \\
Body mass index $\left[\mathrm{kg} / \mathrm{m}^{2}\right]$ & $28.01 \pm 4.6$ & $28.23 \pm 4.9$ & 0.67 \\
Basal LVEF [\%] & $65.5 \pm 5.4$ & $67.4 \pm 6.0$ & 0.75 \\
Hypertension & 35.7 & 29.6 & 0.67 \\
Diabetes & 14.3 & 11.3 & 0.58 \\
Hyperlipidemia & 14.3 & 16.9 & 0.74 \\
Smoking & 42.9 & 32.4 & 0.73 \\
Anthracycline dose $\left[\mathrm{mg} / \mathrm{m}^{2}\right]$ & $243 \pm 4.8$ & $242 \pm 4.6$ & 0.97 \\
Radiotherapy [\%] & 42.9 & 42.3 & 0.08 \\
Radiotherapy total dose [cGy] & 6400.00 & 5823.08 & 0.86 \\
Baseline strain & $18.88 \pm 2.9$ & $18.91 \pm 2.9$ & 0.03 \\
Beta-blockers treatment [\%] & 14 & 0 & 0.68 \\
ACEl treatment [\%] & 7 & 17 & 0.87 \\
Statin treatment [\%] & 14 & 13 & \\
\hline
\end{tabular}

Values are expressed as mean \pm standard deviation; values of $\mathrm{P}$ calculated by Student's $\mathrm{t}$ for the differences in means between groups $\mathrm{AC}+$ and AC. AC + - group of patients who develop anthracycline cardiotoxicity; AC - - group of patients who do not develop cardiotoxicity due to anthracyclines; LVEF — left ventricular ejection fraction; ACEI — angiotensin-converting enzyme inhibitors

Table 3. Echocardiographic parameters at the beginning and the follow-up visits.

\begin{tabular}{lccccc}
\hline & Basal & Visit 1 & Visit 2 & Visit 3 & Visit 4 \\
\hline N (patients) & 85 & 85 & 84 & 83 & 77 \\
LVEF [\%] & $67.13 \pm 5.9$ & $64.74 \pm 5.6^{*}$ & $63.26 \pm 5.1^{*}$ & $64.05 \pm 5.3^{*}$ & $60.89 \pm 7.0^{*}$ \\
GLS [\%] & $18.91 \pm 2.8$ & $18.32 \pm 3.5$ & $17.67 \pm 3.7$ & $17.63 \pm 3.3$ & $18.1 \pm 3.2$ \\
\hline
\end{tabular}

Values expressed as mean \pm standard deviation; *indicates $p$-value $<0.05$ compared to baseline values. The global longitudinal strain (GLS) is expressed in absolute values; LVEF — left ventricular ejection fraction measured by the Simpson method

a mean GLS of $-18.91 \pm 2.8 \%$ (Table 3 ), without differences between the groups divided according to CHT protocols.

At follow-up, a significant decrease was found in LVEF with respect to the baseline visit. At visit 3 (9 months after the last dose of anthracyclines and approximately 1 year after the baseline visit) the mean LVEF by the Simpson method was $64.05 \pm$ $\pm 5.3 \%$, which represents a decrease of $3.08 \%$ with respect to the baseline LVEF (Table 3 ). At this visit, only 1 patient had developed left ventricular systolic dysfunction (1\%). At the end of follow-up, visit 4 (52 months post-CHT) mean LVEF by Simpson method was $60.89 \pm 7.0 \%$, with a significant decrease of $6.24 \%$ with respect to baseline $(\mathrm{p}<0.001$; Table 3); at this visit, another 13 (15\%) patients had developed left ventricular systolic dysfunction. Therefore, it can be seen that most of them appear late in follow-up, and are categorized as late cardiotoxicity. It is remarkable that the decrease in LVEF from visit 3 (9 months after the last dose of CHT) to visit 4 (4 years after the beginning), from $64.05 \%$ to $60.89 \%$, respectively; it can be appreciated how LVEF continues to decrease markedly after the first year (late cardiotoxicity).

Regarding to ventricular diameters, a significant increase in left ventricular diastolic diameter was also detected at visits 3 and 4, and in systolic diameter from visit 1 to visit 3 as well.

In Figure 1 differences are shown in LVEF means between $\mathrm{AC}+$ and $\mathrm{AC}$ - group. There were no significant differences between the $\mathrm{AC}+$ and AC- group from baseline to visit 3 (9 months after the last dose of anthracycline). Significant differences emerge in the last visit, visit 4 (4 years after the start of CHT), showing a mean LVEF of $51.77 \pm 5.15 \%$ in $\mathrm{AC}+$ group and $62.78 \pm 5.82 \%$ in $\mathrm{AC}-$ group $(\mathrm{p}<0.001)$. The greatest percentage 


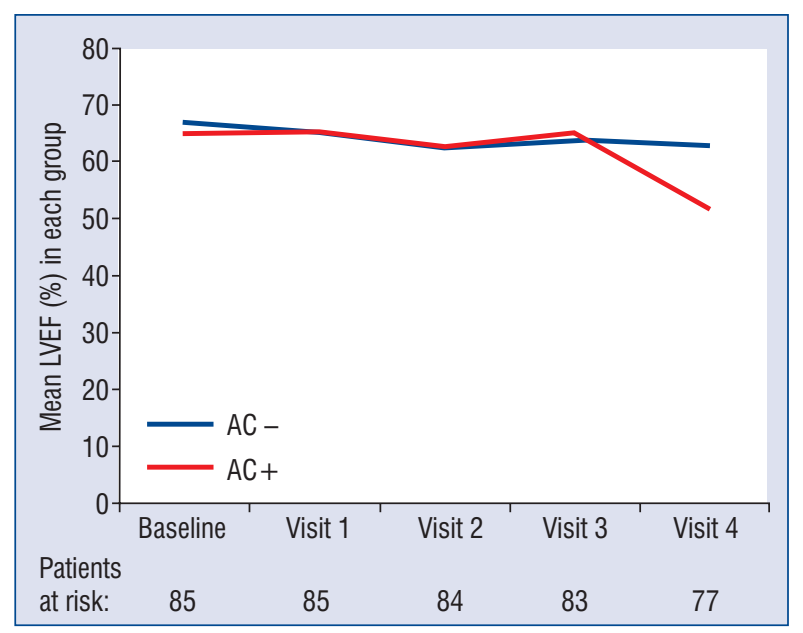

Figure 1. Left ventricular ejection fraction (LVEF) behaviour in $A C+$ group and AC-group, from baseline to final visit. Values expressed as mean \pm standard deviation; $\mathrm{AC}+$ - group of patients who develop anthracycline cardiotoxicity; AC- - group of patients who do not develop cardiotoxicity due to anthracyclines.

decrease in LVEF occurs in $\mathrm{AC}+$ group from visit 3 to visit 4 with a decline of LVEF from $62.78 \%$ to $51.77 \%$, respectively (late cardiotoxicity).

Regarding GLS values (Table 3), a discrete progressive decrease was found throughout the visits, but without becoming a significant difference. In Table 4 the GLS values are shown at each visit in group $\mathrm{AC}+$ and in group $\mathrm{AC}-$.

In Table 4 the GLS values are shown at each visit in group $\mathrm{AC}+$ and in group $\mathrm{AC}-$. Analyzing the decrease in GLS from baseline visit, group $\mathrm{AC}+$ shows a decrease in GLS from the baseline visit to visit 2 (3 months after the last dose of anthracycline CHT) of $2.59 \pm 2.8$ while the group $\mathrm{AC}$ - only shows a decrease of $1.46 \pm 3.6(\mathrm{p}=0.35)$. There are significant differences in the decrease in GLS from the baseline visit to visit 3 ( 9 months after the last dose of anthracycline CHT), the group $\mathrm{AC}+$ shows a decrease of $3.6 \pm 3.21$ while the group $\mathrm{AC}-$ shows only a decrease of $1.41 \pm 3.6(\mathrm{p}=0.04)$.

\section{Discussion}

Despite the benefits of anthracyclines in the treatment of cancer, cardiotoxicity remains a major concern. There are scant data from prospective studies on cardiotoxicity in the current context characterized by regimens that include lower anthracycline doses and include an initial cardiological evaluation aimed to rule out heart disease. The most important results of the present study are that long-term cardiotoxicity due to anthracyclines is high, $16.5 \%$ at 4.5 years, and its highest incidence was late, observed after the first year post-CHT completion in 13 of the 14 patients who developed cardiotoxicity. Fourteen of the 85 patients developed anthracycline cardiotoxicity, but only one had clinical heart failure, 1 patient suffered sudden cardiac death and 12 patients had asymptomatic left ventricular systolic dysfunction. The results of the current study are not consistent with previous incidences reported in retrospective studies, from $5 \%$, when a cumulative lifetime dose of $400 \mathrm{mg} / \mathrm{m}^{2}$ is reached, up to $48 \%$ at $700 \mathrm{mg} / \mathrm{m}^{2}$ [6-8] (including symptomatic and subclinical cardiotoxicity). A prospective study by Cardinale et al. [11] showed an incidence of cardiotoxicity of $9 \%$ in 5 years, which is lower that observed in the current study; a different definition of cardiotoxicity (LVEF decrease $>10$ absolute points, and $<50 \%$ ) may explain the differences in the incidence.

Another important finding was that cardiotoxicity presentation was mostly subclinical. Regarding subclinical cardiotoxicity, a systematic review [21] describes a wide variation in the incidence of long-term asymptomatic cardiac dysfunction between different studies, with incidences ranging

Table 4. Global longitudinal strain decreases from baseline value at each visit in the AC+ group and AC- group.

\begin{tabular}{|c|c|c|c|}
\hline & $A C+$ & $\mathrm{AC}-$ & $\mathbf{P}$ \\
\hline $\mathrm{N}$ & 14 & 71 & \\
\hline Decrease from baseline to visit 1 & $2.00 \pm 3.3$ & $0.98 \pm 2.8$ & 0.31 \\
\hline Decrease from baseline to visit 2 & $2.59 \pm 2.8$ & $1.46 \pm 3.6$ & 0.35 \\
\hline Decrease from baseline to visit 3 & $3.6 \pm 3.2$ & $1.41 \pm 3.6$ & 0.04 \\
\hline Decrease from baseline to visit 4 & $3.10 \pm 2.5$ & $0.76 \pm 3.3$ & 0.04 \\
\hline
\end{tabular}

Values expressed as mean \pm standard deviation; $\mathrm{AC}+-$ group of patients who develop anthracycline cardiotoxicity; AC- - group of patients who do not develop cardiotoxicity due to anthracyclines. Values of $\mathrm{P}$ calculated by the Student t-test for the differences in decrease in global longitudinal strain between groups of patients who develop cardiac toxicity by anthracyclines (AC + ) and those who do not develop cardiotoxicity by anthracyclines (AC-) 
from $0 \%$ and $57 \%$ in the 25 studies included. Differences in anthracycline dose between different studies may explain, in part, the wide variation in the incidence of subclinical cardiotoxicity.

Also to be considered, is that data from large clinical trials with trastuzumab in the adjuvant treatment [22-26], with patient populations and doses were more similar to those of the present study. In these studies, in the treatment arms with anthracyclines alone (without associated trastuzumab), the incidence of clinical heart failure ranged between $0.06 \%$ and $2.03 \%$ and that of left ventricular dysfunction between $2.2 \%$ up to $17 \%$, concordant with the present study.

Global longitudinal strain is regarded as an early sign of cardiotoxicity [10]. In the current study, patients who developed AC have a significantly greater decrease in GLS from baseline to visit 3 (9 months after the last dose of anthracycline CHT) than those who did not.

Some previous data showed that cardiotoxicity occurring in almost all cases in the first year after the end of treatment [11]. The data herein, however, indicate that a monitoring limited to the first year would not detect most of the cases of cardiotoxicity. Therefore, longer monitoring may be advisable.

\section{Limitations of the study}

Several limitations warrant mention. First of all is an observational study, at a single center. The echocardiograms were analyzed by a cardiology expert in echocardiography, off-line and blind to the clinical data of the patients. Intra- and interobserver variability was tested with acceptable results and comparable to those of other single institution studies, however some degree of bias in these results cannot be excluded. A reduction in LVEF was observed in some patients who did not fulfil criteria for cardiotoxicity. Although the oncologic diagnosis was the first diagnosis for all patients, the study population included patients with early and advanced cancer disease, and this fact may have influenced survival and development of late-cardiotoxicity of the patients.

\section{Conclusions}

The incidence of long-term cardiotoxicity in patients treated with a low cumulative dose of anthracyclines is high, $16.5 \%$ at 4.5 years, observed in almost all cases after the first year of follow-up. Therefore, long-term monitoring may be advisable.

\section{Acknowledgments and funding sources}

We express our gratitude to Elena Moreno and Nieves Estival, both nurses at Cardiology Department in Hospital Universitario de Fuenlabrada, and to all the nursing staff at the Oncology outpatient facility for their help in the research protocol. This work was supported by unrestricted grants from Red Temática de Enfermedades Cardiovasculares (RECAVA) RD06/0014/002 of the Instituto de Salud Carlos III (Spanish Ministry of Science and Innovation) and from Red Tematica de Investigación Cooperativa en Enfermedades Cardiovasculares (RIC) RD12/0042/0067 of the Instituto de Salud Carlos III (Ministerio de Economía y Competitividad)/Plan Nacional de I1D1I 2008-2011, cofounded both with FEDER (Fondo Europeo de Desarrollo Regional), and by a competitive grant from Section of Heart Failure and Heart Transplant of the Spanish Society of Cardiology.

\section{Conflict of interest: None declared}

\section{References}

1. Early Breast Trialists' Collaborative Group. Effects of chemotherapy and hormonal therapy for early breast cancer on recurrence and 15-year survival: an overview of the randomised trials. Lancet. 2005; 365(9472): 1687-1717, doi: 10.1016/s01406736(05)66544-0.

2. Hershman DL, McBride RB, Eisenberger A, et al. Doxorubicin, cardiac risk factors, and cardiac toxicity in elderly patients with diffuse B-cell non-Hodgkin's lymphoma. J Clin Oncol. 2008; 26(19): 3159-3165, doi: 10.1200/JCO.2007.14.1242, indexed in Pubmed: 18591554.

3. Felker GM, Thompson RE, Hare JM, et al. Underlying causes and long-term survival in patients with initially unexplained cardiomyopathy. N Engl J Med. 2000; 342(15): 1077-1084, doi: 10.1056/NEJM200004133421502, indexed in Pubmed: 10760308.

4. Steinherz LJ, Steinherz PG, Tan CT, et al. Cardiac toxicity 4 to 20 years after completing anthracycline therapy. JAMA. 1991; 266(12): 1672-1677, indexed in Pubmed: 1886191.

5. Von Hoff DD, Layard MW, Basa P, et al. Risk factors for doxorubicininduced congestive heart failure. Ann Intern Med. 1979; 91(5): 710-717, doi: 10.7326/0003-4819-91-5-710, indexed in Pubmed: 496103.

6. Bristow M, Thompson P, Martin R, et al. Early anthracycline cardiotoxicity. Am J Med. 1978; 65(5): 823-832, doi: 10.1016/00029343(78)90802-1.

7. Shan K, Lincoff AM, Young JB. Anthracycline-induced cardiotoxicity. Ann Intern Med. 1996; 125(1): 47-58, doi: 10.7326/00034819-125-1-199607010-00008, indexed in Pubmed: 8644988.

8. Yeh ET, Tong AT, Lenihan DJ, et al. Cardiovascular complications of cancer therapy: diagnosis, pathogenesis and management. Circulation. 2004; 109: 3122-3131.

9. Swain SM, Whaley FS, Ewer MS. Congestive heart failure in patients treated with doxorubicin: a retrospective analysis of three trials. Cancer. 2003; 97(11): 2869-2879, doi: 10.1002/cncr.11407, indexed in Pubmed: 12767102. 
10. Curigliano G, Cardinale D, Dent S, et al. Cardiotoxicity of anticancer treatments: Epidemiology, detection, and management. CA Cancer J Clin. 2016; 66(4): 309-325, doi: 10.3322/caac.21341, indexed in Pubmed: 26919165.

11. Cardinale D, Colombo A, Bacchiani G, et al. Early detection of anthracycline cardiotoxicity and improvement with heart failure therapy. Circulation. 2015; 131(22): 1981-1988, doi: 10.1161/ CIRCULATIONAHA.114.013777, indexed in Pubmed: 25948538.

12. Rowinsky EK, McGuire WP, Guarnieri T, et al. Cardiac disturbances during the administration of taxol. J Clin Oncol. 1991; 9(9): 1704-1712, doi: 10.1200/JC0.1991.9.9.1704, indexed in Pubmed: 1678781.

13. Zamorano JL, Lancellotti P, Rodriguez D, et al. 016 ESC Position Paper on cancer treatments and cardiovascular toxicity developed under the auspices of the ESC Committee for Practice Guidelines: The Task Force for cancer treatments and cardiovascular toxicity of the European Society of Cardiology (ESC). Eur Heart J. 2016; 37(31): 2768-2801.

14. Lang RM, Bierig M, Devereux RB, et al. American Society of Echocardiography's Guidelines and Standards Committee; European Association of Echocardiography. Recommendations for chamber quantification: a report from the American Society of Echocardiography's Guidelines and Standards Committee and the Chamber Quantification Writing Group, developed in conjunction with the European Association of Echocardiography, a branch of the European Society of Cardiology. J Am Soc Echocardiogr. 2005; 18(12): 1440-1463, doi: 10.1016/j.echo.2005.10.005, indexed in Pubmed: 16376782.

15. Jensen BV, Skovsgaard T, Nielsen SL. Functional monitoring of anthracycline cardiotoxicity: a prospective, blinded, long-term observational study of outcome in 120 patients. Ann Oncol. 2002; 13(5): 699-709, doi: 10.1093/annonc/mdf132, indexed in Pubmed: 12075737.

16. Martín M, Esteva FJ, Alba E, et al. Minimizing cardiotoxicity while optimizing treatment efficacy with trastuzumab: review and expert recommendations. Oncologist. 2009; 14(1): 1-11, doi: 10.1634/ theoncologist.2008-0137, indexed in Pubmed: 19147689.

17. Jain D, Ahmad T, Cairo M, et al. Cardiotoxicity of cancer chemotherapy: identification, prevention and treatment. Ann Transl Med. 2017; 5(17): 348, doi: 10.21037/atm.2017.06.35, indexed in Pubmed: 28936442.
18. Lipshultz SE, Colan SD, Gelber RD, et al. Late cardiac effects of doxorubicin therapy for acute lymphoblastic leukemia in childhood. N Engl J Med. 1991; 324(12): 808-815, doi: 10.1056/ NEJM199103213241205, indexed in Pubmed: 1997853.

19. Lipshultz SE, Lipsitz SR, Sallan SE, et al. Chronic progressive cardiac dysfunction years after doxorubicin therapy for childhood acute lymphoblastic leukemia. J Clin Oncol. 2005; 23(12): 2629-2636, doi: 10.1200/JCO.2005.12.121, indexed in Pubmed: 15837978.

20. Russell RR, Alexander J, Jain D, et al. The role and clinical effectiveness of multimodality imaging in the management of cardiac complications of cancer and cancer therapy. J Nucl Cardiol. 2016; 23(4): 856-884, doi: 10.1007/s12350-016-0538-8, indexed in Pubmed: 27251147.

21. Kremer LCM, van der Pal HJH, Offringa M, et al. Frequency and risk factors of subclinical cardiotoxicity after anthracycline therapy in children: a systematic review. Ann Oncol. 2002; 13(6): 819-829, doi: 10.1093/annonc/mdf167, indexed in Pubmed: 12123328.

22. Moja L, Tagliabue L, Balduzzi S, et al. Regímenes con trastuzumab para el cáncer de mama temprano. Cochrane Database Syst Rev. 2012: CD006243.

23. Suter TM, Procter M, van Veldhuisen DJ, et al. Trastuzumabassociated cardiac adverse effects in the herceptin adjuvant trial. J Clin Oncol. 2007; 25(25): 3859-3865, doi: 10.1200/ JC0.2006.09.1611, indexed in Pubmed: 17646669.

24. Piccart-Gebhart M, Procter M, Leyland-Jones B, et al. Trastuzumab after Adjuvant Chemotherapy in HER2-Positive Breast Cancer. New Engl J Med. 2005; 353(16): 1659-1672, doi: 10.1056/nejmoa052306.

25. Romond EH, Perez EA, Bryant J, et al. Trastuzumab plus adjuvant chemotherapy for operable HER2-positive breast cancer. N Engl J Med. 2005; 353(16): 1673-1684, doi: 10.1056/NEJMoa052122, indexed in Pubmed: 16236738.

26. Tan-Chiu E, Yothers G, Romond E, et al. Assessment of cardiac dysfunction in a randomized trial comparing doxorubicin and cyclophosphamide followed by paclitaxel, with or without trastuzumab as adjuvant therapy in node-positive, human epidermal growth factor receptor 2-overexpressing breast cancer: NSABP B-31. J Clin Oncol. 2005; 23(31): 7811-7819, doi: 10.1200/ JC0.2005.02.4091, indexed in Pubmed: 16258083. 